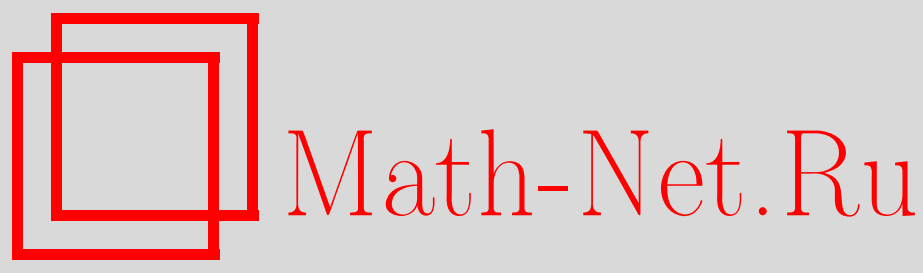

И. Хен, М. Карлинер, Решеточная структура бэйбискирмионов, ТМФ, 2009, том 160, номер 1, 69-83

DOI: https://doi.org/10.4213/tmf6379

Использование Общероссийского математического портала Math-Net.Ru подразумевает, что вы прочитали и согласны с пользовательским соглашением http: //www . mathnet.ru/rus/agreement

Параметры загрузки:

IP : 54.80 .97 .219

26 апреля 2023 г., $17: 16: 28$

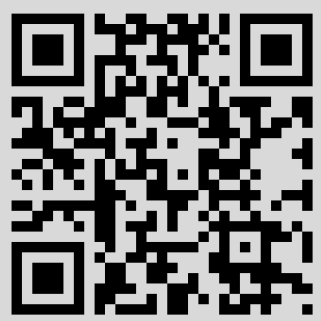




\section{РЕШЕТОЧНАЯ СТРУКТУРА БЭЙБИ-СКИРМИОНОВ}

Исследуется решеточная структура бэйби-скирмионов при нулевой температуре с использованием численного алгоритма, производящего минимизацию по всем полям. Бэйби-скирмионы помещаются внутрь различных единичных ячеек, имеющих форму параллелограмма, после чего налагаются периодические граничные условия. Получено, что минимальная энергия достигается на шестиугольной решетке и что в данной конфигурации скирмион расщепляется на четверть-скирмионы. В частности, энергия в случае шестиугольной решетки оказывается ниже, чем в хорошо исследованном случае прямоугольной решетки, где наблюдается расщепление на полускирмионы.

Ключевые слова: бэйби-скирмион, скирмион, кристаллическая структура.

\section{1. ВВЕДЕНИЕ}

Модель Скирма [1] представляет собой $S U(2)$-значную нелинейную теорию адронов в размерности $3+1$ с топологическими солитонными решениями, называемыми скирмионами. Кроме кинетического члена, лагранжиан модели содержит "скирмионный” член, имеющий четвертый порядок по производным и вводящий в модель масштабный параметр [2]. Существование устойчивых решений в модели Скирма является следствием нетривиальной топологии отображений $\mathcal{M}$ физического пространства в пространство полей при фиксированном времени, $\mathcal{M}: S^{3} \rightarrow S U(2) \cong S^{3}$, где физическое пространство $\mathbb{R}^{3}$ компактифицируется до $S^{3}$ с помощью наложения требования эквивалентности всех направлений на пространственной бесконечности. Топология, которая возникает из этой одноточечной компактификации, позволяет объединить эти отображения в классы эквивалентности, в каждом из которых имеется единственная сохраняющаяся величина, называемая топологическим зарядом.

В размерности $2+1$ модель Скирма имеет аналог, известный как бэйби-модель Скирма; она также допускает устойчивые полевые конфигурации солитонной природы [3]. Из-за меньшей размерности эта модель представляет собой упрощенный вариант исходной модели; тем не менее она имеет и самостоятельное физическое значение, связанное с несколькими приложениями к системам с квантовым эффектом

${ }^{*}$ Raymond and Beverly Sackler School of Physics and Astronomy, Tel-Aviv University, Tel-Aviv, Israel. E-mail: itayhe@post.tau.ac.il, marek@proton.tau.ac.il 
Холла, в которых бэйби-скирмионы возникают как низкоэнергетические возбуждения основного состояния вблизи ферромагнитных чисел заполнения [4]-[9].

Таргет-многообразие в бэйби-модели Скирма описывается трехмерным вектором $\boldsymbol{\phi}=\left(\phi_{1}, \phi_{2}, \phi_{3}\right)$, на который наложена связь $\boldsymbol{\phi} \cdot \boldsymbol{\phi}=1$. По аналогии с $(3+1)$-мерным случаем область $\mathbb{R}^{2}$ определения этой модели компактифицируется до $S^{2}$, что приводит к топологии, на основании которой можно разбить полевые конфигурации этой модели на классы с сохраняющимися топологическими зарядами. Плотность лагранжиана бэйби-модели Скирма состоит из кинетического, скирмионного и потенциального членов:

$$
\mathcal{L}=\frac{1}{2} \partial_{\mu} \boldsymbol{\phi} \cdot \partial^{\mu} \boldsymbol{\phi}-\frac{\kappa^{2}}{2}\left[\left(\partial_{\mu} \boldsymbol{\phi} \cdot \partial^{\mu} \boldsymbol{\phi}\right)^{2}-\left(\partial_{\mu} \boldsymbol{\phi} \cdot \partial_{\nu} \boldsymbol{\phi}\right) \cdot\left(\partial^{\mu} \boldsymbol{\phi} \cdot \partial^{\nu} \boldsymbol{\phi}\right)\right]-U\left(\phi_{3}\right) .
$$

Если в размерности $3+1$ последнее слагаемое можно включать или не включать [10], то в $(2+1)$-мерной модели его присутствие необходимо для устойчивости решений. Однако за исключением требования обращения потенциала в нуль на бесконечности для заданного вакуумного значения поля (обычно выбираемого в виде $\left.\phi^{(0)}=(0,0,1)\right)$ точный вид потенциала является произвольным, что приводит к богатому семейству возможных бэйби-моделей Скирма.

В литературе подробно исследовались модели с несколькими конкретными потенциалами [3], [11]-[17]. Простейшей является голоморфная модель, в которой $U\left(\phi_{3}\right)=\mu^{2}\left(1-\phi_{3}\right)^{4}$, при этом известно, что устойчивые решения имеются только в секторе с зарядом единица [11]-[13]. Широко изучалась также модель с потенциалом $U\left(\phi_{3}\right)=\mu^{2}\left(1-\phi_{3}\right)$ (обычно называемая "старой” моделью). Этот потенциал приводит к сильно структурированным устойчивым мультискирмионам без вращательной симметрии [3], [14]. Для однопараметрического семейства потенциалов $U=\mu^{2}\left(1-\phi_{3}\right)^{s}$ недавно были найдены многосолитонные решения, которые дают интерполяцию между "старым" и голоморфным потенциалами [17].

В секторе с зарядом единица устойчивые решения с вращательной симметрией существуют при всех значениях $s$, но скирмионная энергия как функция $s$ не монотонна: она достигает минимума при $s \approx 2.2$ (см. рис. 1 ).

В секторе с зарядом два также было установлено существование устойчивых решений, но только при $s<2$. Они имеют вращательную симметрию и подобны кольцам, что соответствует двум скирмионам с зарядом единица, расположенным один над другим. Как и для скирмиона с зарядом единица, энергия скирмиона с зарядом два также имеет минимум по $s$ при $s \approx 1.3$. Как показано на рис. 1, при $s \approx 2$ энергия кольцеподобной конфигурации достигает значения удвоенной энергии скирмиона с зарядом единица, и устойчивые конфигураци более не существуют. Начиная с этого момента скирмион распадается на составляющие его скирмионы с зарядом единица, которые в свою очередь начинают удаляться друг от друга. Контурные графики распределения энергии для скирмиона с зарядом два показаны на рис. 2 для двух значений параметра $s$. На этом и последующих контурных рисунках чем больше значение величины, отображаемой на графике, тем светлее тон. Если при $s=1.5$ существует кольцеподобная устойчивая конфигурация (см. рис. 2а), то при 


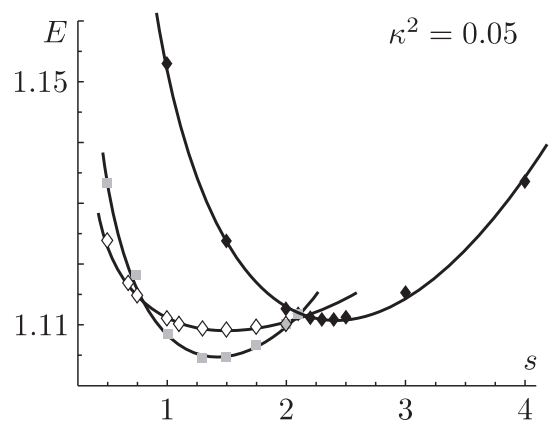

a

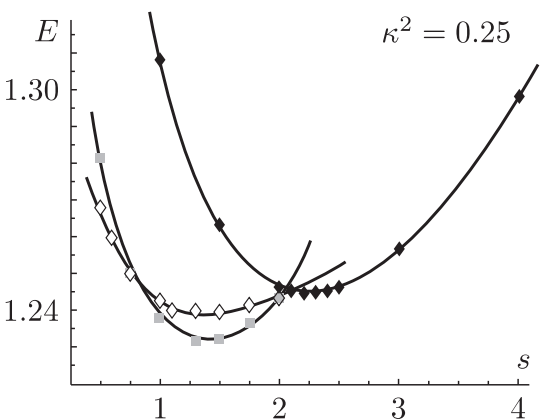

6

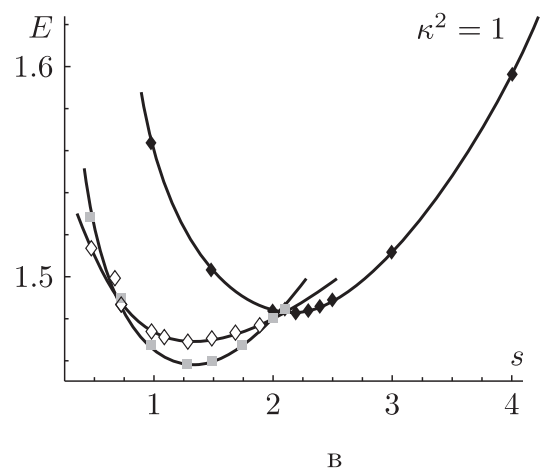

Рис. 1. Полная энергия (деленная на $4 \pi B$ ) скирмионов с зарядом единица $(\boldsymbol{\vee})$, с зарядом два $(\boldsymbol{\square})$ и с зарядом три $(\diamond)$ как функция параметра $s$ потенциала $U$ при разных значениях $\kappa\left(\mu^{2}=0.1\right)$. Каждый из графиков энергии имеет минимум при некотором $s$. При $s \approx 2$ энергия на единицу топологического заряда для решений с зарядом два и зарядом три достигает (снизу) энергии решения с зарядом единица, и устойчивых решений более не наблюдается.

$s=2.6$ скирмион распадается на части. Последний случай показан на рис. 2б, который соответствует продолжающемуся увеличению расстояния между отдельными скирмионами.

Как и в случае скирмионов с зарядом два, существование устойчивых скирмионов с зарядом три также зависит от $s$. При значениях выше $s \approx 2$ не существует устойчивых решений с зарядом три; энергия скирмиона становится больше, чем утроенная энергия скирмиона с зарядом единица, и устойчивые конфигурации более не реализуются. Это показано на рис. 3, где приведены контурные графики распределения энергии для скирмиона с зарядом три при разных значениях $s$. При $s=0.5$ (рис. 3а) решение имеет вращательную симметрию, а при $s=0.75$ и $s=1$ (рис. 3б и 3в соответственно) вращательная симметрия решения нарушена. При $s=3$ устойчивых решений не существует. Последний случай показан на рис. Зг, 


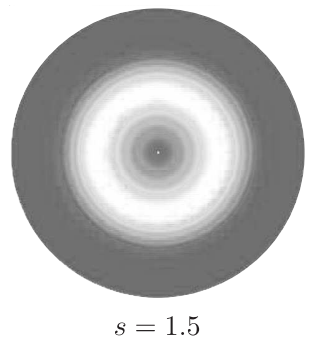

a

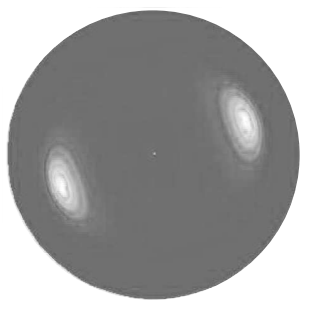

$s=2.6$

б

Рис. 2. Контурные графики распределений энергии для скирмиона с зарядом два $\left(\kappa^{2}=1, \mu^{2}=0.1\right)$. При $s<2$ существуют кольцеподобные конфигурации с вращательной симметрией, соответствующие двум скирмионам с зарядом единица, расположенным один над другим (а). При $s>2$ скирмион с зарядом два распадается на два скирмиона с зарядом единица, расходящиеся на бесконечное расстояние (б).

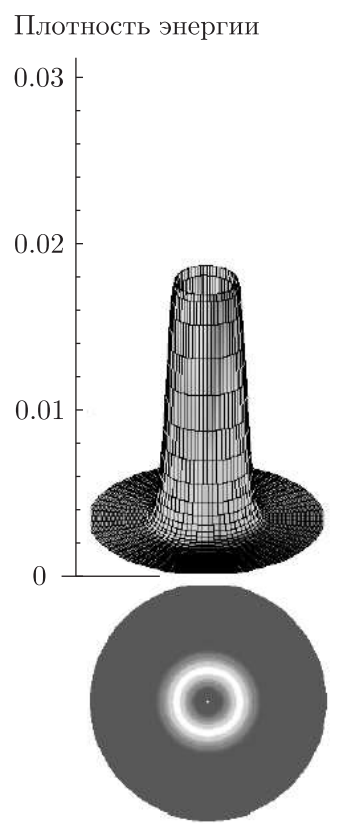

$s=0.5$

a

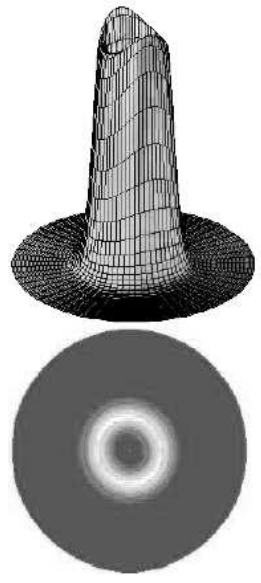

$s=0.75$

6

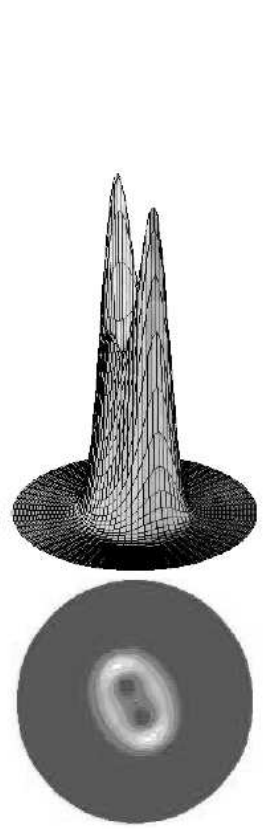

$s=1$

B

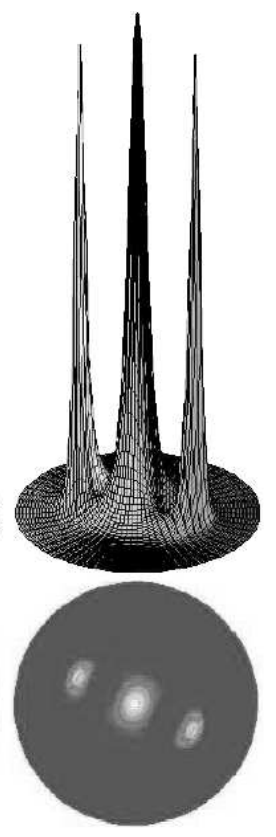

$s=3$

$\Gamma$

Рис. 3. Плотности энергии и контурные графики для скирмионов с зарядом три при различных $s\left(\kappa^{2}=0.01, \mu^{2}=0.1\right)$. При $s=0.5$ (а) конфигурация с минимальной энергией вращательно симметрична, что соответствует трем скирмионам с зарядом единица, расположенным один над другим. При $s=0.75$ (б) и $s=1$ (в) решения демонстрируют только $\mathbb{Z}(2)$-симметрию, что соответствует частично перекрывающимся скирмионам с зарядом единица. При $s=3$ (г) устойчивых решений не существует. 
он соответствует дальнейшему увеличению расстояния между отдельными скирмионами. Энергия скирмиона с зарядом три также имеет минимум по $s$ при $s \approx 1.5$ (см. рис. 1).

Итак, установлено, что точный вид потенциального члена в бэйби-модели Скирма оказывает решающее влияние на существование и устойчивость решений. В частности, показатель в потенциальном члене (параметр $s$ ) играет роль "управляющего" параметра для интенсивности притяжения (малые значения $s$ ) или отталкивания (большие значения $s$ ) между отдельными скирмионами.

\section{2. РЕШЕТОЧНАЯ СТРУКТУРА БЭЙБИ-СКИРМИОНОВ}

В данном разделе мы следуем работе [18]. В качестве низкоэнергетической эффективной теории адронов модель Скирма использовалась для описания систем из нескольких нуклонов, кроме того, она применялась при рассмотрении ядерной и кварковой материи [19]-[21]. Одним из наиболее сложных аспектов физики адронов является поведение фазовой диаграммы адронной материи при конечной плотности и низкой или даже нулевой температуре. Свойства скирмионов на решетке при нулевой температуре представляют интерес отчасти ввиду того, что поведение ядерной материи при больших плотностях в настоящее время привлекает к себе значительное внимание. При описании в рамках стандартной модели Скирма при нулевой температуре кристалл нуклонов превращается в кристалл полунуклонов при конечной плотности [22]-[26].

Для исследования скирмионных кристаллов на поле Скирма налагают периодические граничные условия и работают в пределах единичной ячейки (см. книгу [10]). В работе [22] была предпринята первая попытка построения кристалла с использованием простой кубической решетки скирмионов, симметрии которой делают максимальным притяжение между ближайшими соседями. Предлагались и другие симметрии, приводящие к несколько меньшей, но не минимальной энергии кристаллов [23], [24]. В настоящее время понятно, что лучше всего исходно расставить скирмионы в гранецентрированную кубическую решетку, а их ориентации выбрать симметричными, чтобы обеспечить максимальное притяжение между всеми ближайшими соседями [25], [26].

Бэйби-модель Скирма изучалась также в связи со скирмионными решетками различной структуры [27]-[29]; известно также, что бэйби-скирмионы расщепляются на полускирмионы, когда их помещают внутри прямоугольной решетки [29]. Однако к настоящему моменту не известно, приводят ли в прямоугольнике периодические граничные условия к конфигурациям с энергией, минимальной по всем возможным типам решеток, или же имеются другие, непрямоугольные бэйби-скирмионные решеточные конфигурации, которые имеют меньшую энергию. Поиск ответов на эти вопросы особенно важен как ввиду их актуальности для систем с квантовым эффектом Холла в двумерном пространстве, так и потому, что их можно использовать при построении гипотез о кристаллической структуре нуклонов в трехмерном пространстве. 
Ниже мы найдем кристаллическую структуру бэйби-скирмионов, помещая их внутри различных параллелограммов с периодическими граничными условиями, при этом будем искать конфигурации с энергией, минимальной по всем параллелограммам с фиксированной площадью (тем самым плотность скирмионов будет оставаться фиксированной) [30]. Мы покажем, что существует параллелограмм определенного типа, а именно отвечающий шестиугольной решетке, для которого конфигурация имеет минимальную энергию. В частности, эта энергия ниже, чем энергия известной конфигурации из квадратных ячеек, в которой скирмион расщепляется на полускирмионы. Как будет указано ниже, обнаруженная нами шестиугольная структура не уникальна для данной модели, но возникает и в других солитонных моделях, таких как вихри Гинзбурга-Ландау [31], системы с квантовым эффектом Холла [5], [6] и даже в контексте трехмерных скирмионов [32]. Причина такого положения дел обсуждается в заключительном разделе.

\section{3. БЭЙБИ-СКИРМИОНЫ ВНУТРИ ПАРАЛЛЕЛОГРАММА}

Статическими решениями бэйби-модели Скирма являются те полевые конфигурации, которые в каждом топологическом секторе минимизируют функционал энергии

$$
E=\frac{1}{2} \int_{\Lambda} d x d y\left(\left(\partial_{x} \boldsymbol{\phi}\right)^{2}+\left(\partial_{y} \phi\right)^{2}+\kappa^{2}\left(\partial_{x} \boldsymbol{\phi} \times \partial_{y} \phi\right)^{2}+2 \mu^{2}\left(1-\phi_{3}\right)\right) .
$$

Это можно получить непосредственно в случае плотности лагранжиана (1), где используется потенциал “старой” модели. В нашем подходе интегрирование ведется по параллелограммам

$$
\Lambda=\left\{\alpha_{1}(L, 0)+\alpha_{2}(s L \sin \gamma, s L \cos \gamma) ; \quad 0 \leqslant \alpha_{1}, \alpha_{2}<1\right\},
$$

где $L$ - длина одной стороны параллелограмма, $s L$ при $0<s \leqslant 1$ - длина другой его стороны, а $0 \leqslant \gamma<\pi / 2$ - угол между стороной длины $s L$ и нормалью к стороне длины $L$. Каждый параллелограмм задается, таким образом, набором параметров $\{L, s, \gamma\}$, а скирмионная плотность внутри параллелограмма есть $\rho=B /\left(s L^{2} \cos \gamma\right)$, где $B$ - топологический заряд скирмиона. Периодические граничные условия учитываются за счет отождествления каждой из двух противоположных сторон параллелограмма:

$$
\boldsymbol{\phi}(\mathbf{x})=\boldsymbol{\phi}\left(\mathbf{x}+n_{1}(L, 0)+n_{2}(s L \sin \gamma, s L \cos \gamma)\right),
$$

где $n_{1}, n_{2} \in \mathbb{Z}$. Нас интересуют статические решения с конечной энергией, которые на языке дифференциальной геометрии являются отображениями $\mathbb{T}^{2} \mapsto S_{2}$, где $\mathbb{T}^{2}$ - двумерный тор. Они разбиваются на гомотопические секторы, параметризованные инвариантным интегралом топологического заряда $B$ - степенью отображения, определяемой как

$$
B=\frac{1}{4 \pi} \int_{\Lambda} d x d y\left(\phi \cdot\left(\partial_{x} \phi \times \partial_{y} \phi\right)\right) .
$$

Можно показать, что статическая энергия $E$ удовлетворяет условию

$$
E \geqslant 4 \pi B
$$


причем равенство возможно только в “чистом" $O(3)$-случае (т.е. когда и скирмионнный, и потенциальный члены отсутствуют) [29]. Заметим, что если в бэйби-модели Скирма на $\mathbb{R}^{2}$ при фиксированных граничных условиях потенциальный член необходим для предотвращения неограниченного увеличения солитонов, то в нашем формализме в силу периодических граничных условий этого не требуется [29]. В настоящей работе мы исследуем модель как с потенциальным членом, так и без него.

Рассматриваемую задачу можно упростить с помощью линейного отображения параллелограммов $\Lambda$ в двумерный тор $\mathbb{T}^{2}$ с единичной площадью. В новых координатах функционал энергии принимает вид

$$
\begin{gathered}
E=\frac{1}{2 s \cos \gamma} \int_{\mathbb{T}^{2}} d x d y\left(s^{2}\left(\partial_{x} \phi\right)^{2}-2 s \sin \gamma\left(\partial_{x} \phi \partial_{y} \phi\right)+\left(\partial_{y} \phi\right)^{2}\right)+ \\
+\frac{\kappa^{2} \rho}{2 B} \int_{\mathbb{T}^{2}} d x d y\left(\partial_{x} \boldsymbol{\phi} \times \partial_{y} \boldsymbol{\phi}\right)^{2}+\frac{\mu^{2} B}{\rho} \int_{\mathbb{T}^{2}} d x d y\left(1-\phi_{3}\right) .
\end{gathered}
$$

Заметим, что энергия зависит от параметров модели Скирма $\kappa$ и $\mu$ и скирмионной плотности $\rho$ только через комбинации $\kappa^{2} \rho$ и $\mu^{2} / \rho$.

Чтобы найти конфигурации скирмионов с энергией, минимальной по всем параллелограммам с фиксированной площадью (или, что эквивалентно, с заданной плотностью $\rho$ ), мы перебираем значения параметров параллелограммов $\{s, \gamma\}$ и находим параллелограмм, для которого получающаяся энергия минимальна по всему пространству параметров. Мы применяем также альтернативный подход к данной задаче, несколько более аналитический по своей природе; он подробно рассматривается в разделе 6 . В следующем разделе дано описание процедуры численной минимизации.

\section{4. ПРОЦЕДУРА ЧИСЛЕННОЙ МИНИМИЗАЦИИ}

Уравнения Эйлера-Лагранжа, выводимые из функционала энергии (7), являются нелинейными дифференциальными уравнениями в частных производных, так что, вообще говоря, для их решения требуются численные методы. Мы фиксировали заряд $B$, параметры $s, \gamma$ параллелограмма и скирмионную плотность $\rho$, после чего искали скирмионную конфигурацию с минимальной энергией с помощью метода полной релаксации полей на прямоугольной сетке размера $100 \times 100$ на двумерном торе $\mathbb{T}^{2}$, где в каждой точке был определен триплет полей $\phi\left(\chi_{m}, y_{m}\right)$. Для вычисления плотностей энергии и заряда использовалась процедура, разработанная в работе [33], в рамках которой расчет этих величин выполнялся в центрах квадратов сетки. Численные производные также вычислялись в этих точках; $x$-производные рассчитывались по формуле

$$
\begin{aligned}
\left.\frac{\partial \phi}{\partial x}\right|_{\left(x_{m+1 / 2}, y_{n+1 / 2}\right)}=\frac{1}{\Delta x} & \left(\left\langle\frac{\phi\left(x_{m+1}, y_{n}\right)+\phi\left(x_{m+1}, y_{n+1}\right)}{2}\right\rangle_{\text {norm. }}-\right. \\
& \left.-\left\langle\frac{\phi\left(x_{m}, y_{n}\right)+\phi\left(x_{m}, y_{n+1}\right)}{2}\right\rangle_{\text {norm. }}\right)
\end{aligned}
$$

где индекс "norm" указывает, что усредненные поля нормированы на единицу; $y$-производные рассчитывались аналогично. Мы обнаружили, что эта процедура 
прекрасно работает. По поводу более подробного обсуждения данного метода см. работу [33].

Процесс релаксации начинается с задания триплета полей $\phi$ с вращательно-симметричной конфигурацией

$$
\phi_{\text {init }}=(\sin f(r) \cos B \theta, \sin f(r) \sin B \theta, \cos f(r)) \text {, }
$$

где функция профиля $f(r)=\pi e^{-r}$, а $r, \theta$ - обычные полярные координаты. Далее энергия бэйби-скирмиона минимизируется путем повторения следующих шагов: на сетке случайным образом выбирается точка $\left(x_{m}, y_{n}\right)$ вместе с одной из трех компонент поля $\phi\left(x_{m}, y_{n}\right)$. Выбранная компонента затем сдвигается на значение $\delta_{\phi}$, меняющееся равномерно на интервале $\left[-\Delta_{\phi}, \Delta_{\phi}\right]$, где исходно $\Delta_{\phi}=0.1$. Затем триплетное поле нормируется и вычисляется изменение энергии. Если энергия убывает, то модифицикация поля принимается, в противном случае она отбрасывается. Затем выбирается меньшее значение $\Delta_{\phi}$ и процедура повторяется до тех пор, пока дальнейшее уменьшение энергии не перестанет наблюдаться.

Чтобы проверить устойчивость и надежность нашей численной процедуры, мы запускали минимизационный алгоритм, используя различные начальные конфигурации и сетку размера $80 \times 80$ для нескольких значений $\rho, s$ и $\gamma$. Это делалось с целью удостовериться, что конечные конфигурации не зависят от дискретизации и от выбранной схемы охлаждения. В качестве дальнейшей проверки мы повторяли некоторые из процедур минимизации, вычисляя численные производные с более высокой точностью, с использованием восьми точек поля. Никаких видимых изменений в результатах обнаружено не было.

\section{5. РЕЗУЛЬТАТЫ РАСЧЕТОВ}

Используя процедуру минимизации, описанную в предыдущем разделе, мы нашли статические скирмионные конфигурации с энергией, минимальной по всем параллелограммам, для различных конфигураций: в “чистом" $O(3)$-случае, когда и параметр Скирма $\kappa$, и потенциал взаимодействия $\mu$ полагаются равными нулю, в случае Скирма, когда только $\mu=0$, и в общем случае, когда ни скирмионный член, ни потенциальный член не равны нулю.

В каждой из этих конфигураций производился перебор в пространстве параметров параллелограммов при фиксированной скирмионной плотности $\rho$, что при каждом наборе $\{s, \gamma\}$ приводило к некоторой конфигурации с минимальной энергией. Выбор числа скирмионов, которые следует разместить внутри единичной ячейки, делался после некоторого предварительного тестирования, в ходе которого исследовались также скирмионы с другими зарядами (до $B=8$ ). Оказалось, что конфигурации с минимальной энергией с нечетными зарядами имеют существенно бо́льшие энергии, чем конфигурации с четными зарядами, причем среди последних наиболее фундаментальным оказался скирмион с зарядом два, поскольку было видно, что он служит "строительным элементом" для скирмионов с бо́льшими зарядами. Ниже представлена сводка результатов. 


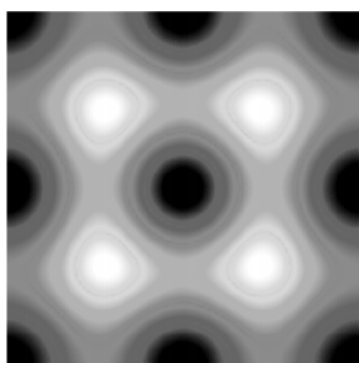

$s=1, \gamma=0$

a

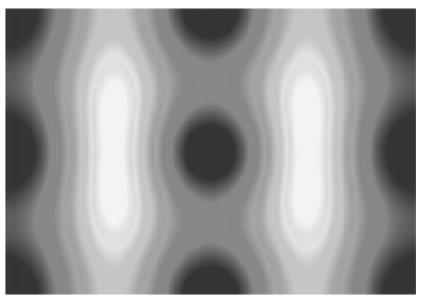

$s=0.7, \gamma=0$

$\mathrm{B}$

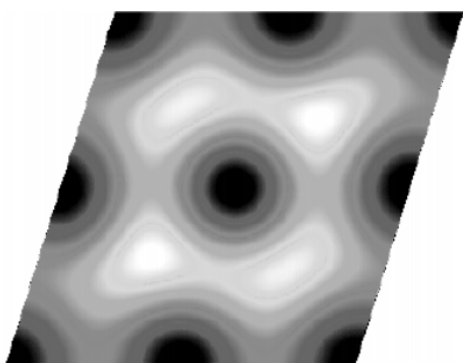

$s=0.9, \gamma=\pi / 16$

б

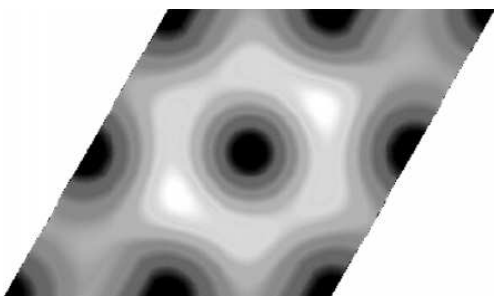

$s=1, \gamma=\pi / 6$

$\Gamma$

Рис. 4. Скирмионы с зарядом два в чистом $O(3)$-случае: контурные графики зарядовых плотностей для различных конфигураций параллелограммов, из которых все дают минимальное значение в ограничении на энергию (6): $E=4 \pi B=8 \pi$.

5.1. Чистый $O(3)$-случай $(\kappa=\mu=0)$. Чистый $O(3)$-случай соответствует ситуации, когда и $\kappa$, и $\mu$ равны нулю. В этом случае можно найти аналитические решения в терминах эллиптических функций Вейерштрасса [29], а конфигурациям с энергией, минимальной по всем параллелограммам, отвечает предельный случай равенства в ограничении на энергию (6), т.е. для них $E=4 \pi B$. Таким образом, сравнение численных результатов с аналитическими решениями служит полезной проверкой точности нашей численной процедуры. Наблюдается согласие с точностью до шести значащих цифр. Контурные графики зарядовых плотностей для различных параллелограммов в случае скирмионов с зарядом два показаны на рис. 4, все они имеют энергию $E=8 \pi$.

5.2. Случай Скирма $(\kappa \neq 0, \mu=0)$. Как отмечалось выше, при $\mu=0$ функционал энергии зависит от параметра Скирма $\kappa$ только через $\kappa^{2} \rho$, так что без потери общности можно варьировать $\rho$, держа при этом фиксированным $\kappa^{2}=0.03$ (такой специальный выбор $\kappa$ был сделан для удобства численных расчетов). На рис. 5 показаны полные энергии (деленные на $8 \pi$ ) и соответствующие контурные графики зарядовых плотностей для шестиугольной, квадратной и других конфигураций (для сравнения), все при $\rho=2$. Минимизация функционала энергии (7) по всем 


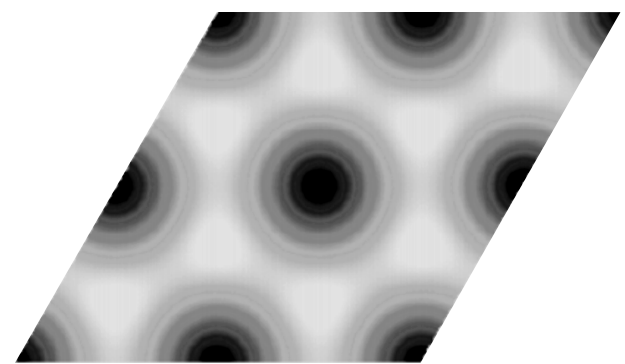

$s=1, \gamma=\pi / 6, E / 8 \pi=1.433$

a

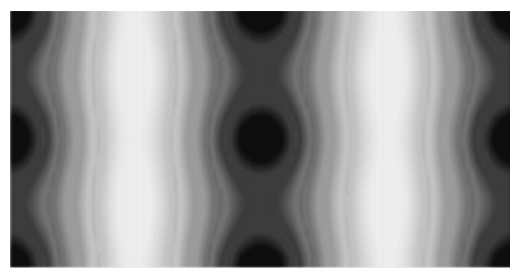

$s=0.51, \gamma=0, E / 8 \pi=1.587$

B

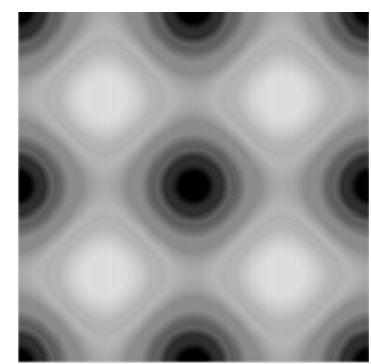

$s=1, \gamma=0, E / 8 \pi=1.446$ 6

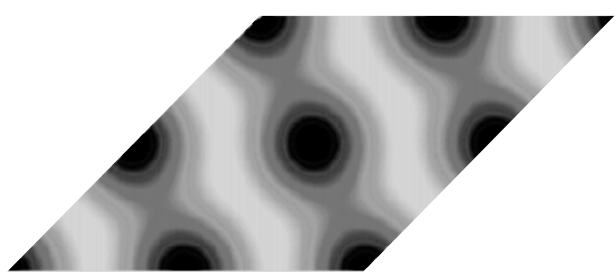

$s=1, \gamma=\pi / 4, E / 8 \pi=1.454$

$\Gamma$

Рис. 5. Скирмионы с зарядом два в случае Скирма при $\kappa^{2}=0.03$ и $\rho=2$ : контурные графики зарядовых плотностей для шестиугольной, квадратной и других фундаментальных ячеек. Шестиугольная конфигурация имеет наименьшую энергию.

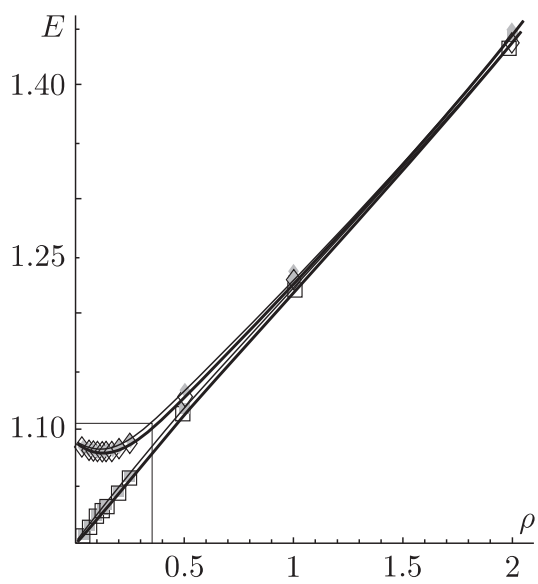

a

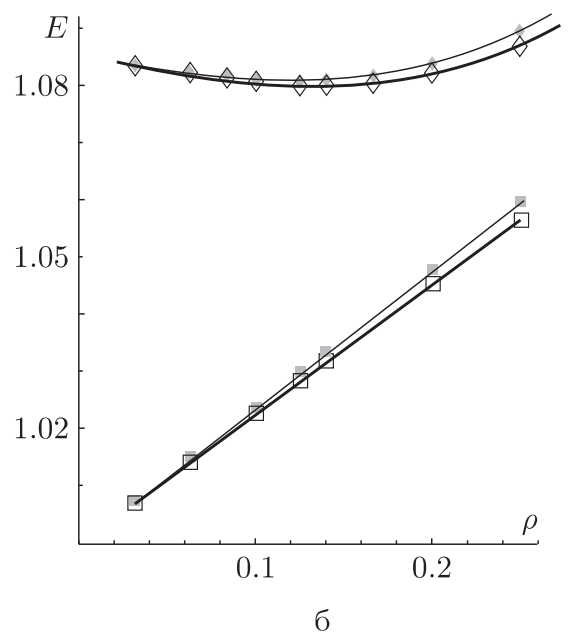

Рис. 6. Зависимости полной энергии $E$ (деленной на $8 \pi$ ) скирмиона с зарядом два от скирмионной плотности в шестиугольной решетке ( $\square$ - случай Скирма и $\diamond$ - общий случай) и в квадратной решетке ( - случай Скирма и - общий случай), в случае Скирма $\kappa^{2}=0.03$, в общем случае $\kappa^{2}=0.03$ и $\mu^{2}=0.1$. В общем случае существует оптимальная плотность (при $\rho \approx 0.14$ ), при которой энергия достигает глобального минимума. Рисунок "б” представляет собой увеличенный нижний левый фрагмент на рисунке "а". 
параллелограммам дает следующее. Для любой фиксированной плотности $\rho$ минимальная энергия получается при $s=1$ и $\gamma=\pi / 6$. Этот набор значений соответствует "шестиугольной" решетке, т.е. решетке из "равносторонних треугольников." В этой конфигурации любые три соседних местоположения с нулевой энергией (или "дыры") являются вершинами равносторонних треугольников и наблюдаются восемь различных пиков высокой плотности (как показано на рис. 5а). Эту конфигурацию можно, таким образом, интерпретировать как расщепление два-скирмиона на восемь четверть-скирмионов, причем этот результат не зависит от скирмионной плотности $\rho$. Оказалось, что хорошо исследованная конфигурация квадратных ячеек с минимальной энергией (рис. 5б), в которой два-скирмион расщепляется на четыре полускирмиона, имеет более высокую энергию, чем в случае шестиугольных ячеек.

Полная энергия скирмионов в случае шестиугольных ячеек оказалась пропорциональной плотности скирмионов, что отражает масштабную инвариантность модели (рис. 6). В частности, глобальный минимум энергии $E=4 \pi B=8 \pi$ достигается при $\rho \rightarrow 0$. Это находится в согласии с ожиданиями, поскольку случай $\rho=0$ эквивалентен случаю с нулевым параметром Скирма $\kappa$, а тогда данная модель эффективно является чистой $O(3)$-моделью, и неравенство (6) обращается в равенство.

5.3. Общий случай $(\kappa \neq 0, \mu \neq 0)$. Оказалось, что шестиугольная структура энергетически предпочтительна также и в общем случае. Поскольку, однако, в этом случае скирмионы обладают определенным размером (в чем можно удостовериться, взглянув на зависимость функционала энергии от $\rho$ ), структура скирмионов различна при низких и высоких плотностях, и наблюдается фазовый переход. Если при низких плотностях скирмионы располагаются изолированно друг от друга, то при высоких плотностях они сливаются, образуя четверть-скирмионный кристалл, как и в описанном выше случае Скирма. Когда плотность $\rho$ убывает или, что эквивалентно, значение $\mu$ возрастает, размер скирмионов становится малым по сравнению с размером ячейки. Точная форма решетки перестает оказывать влияние, и разность энергий для различных типов решеток становится очень малой (см. рис. 6).

Более того, вследствие конечного размера скирмиона имеется оптимальная плотность, при которой энергия минимальна. На рис. 7 показаны контурные графики зарядовой плотности скирмиона с зарядом два для нескольких плотностей при $\kappa^{2}=0.03$ и $\mu^{2}=0.1$. Энергия скирмиона минимальна при $\rho \approx 0.14$ (см. рис. 6 ).

\section{6. ПОЛУАНАЛИТИЧЕСКИЙ ПОДХОД}

Функционал энергии (7) зависит и от поля Скирма $\phi$, и от параметров параллелограмма $\gamma$ и $s$. Формально конфигурацию с минимальной энергией по всем параллелограммам можно получить путем функционального дифференцирования по $\phi$ и обычного дифференцирования по $\gamma$ и $s$. Однако поскольку получающиеся уравнения очень сложны, прямое численное решение весьма затруднительно. Тем не менее некоторых аналитических успехов можно добиться следующим образом. Сначала продифференцируем функционал энергии (7) только по $\gamma$ и $s$ и приравняем частные 


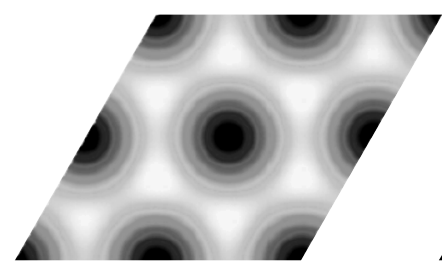

$\rho=1, E / 8 \pi=1.229$

a

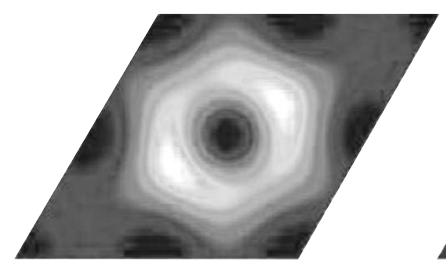

$\rho=0.14, E / 8 \pi=1.08$ $\Gamma$

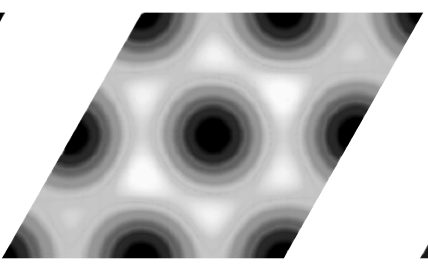

$\rho=0.5, E / 8 \pi=1.128$

б

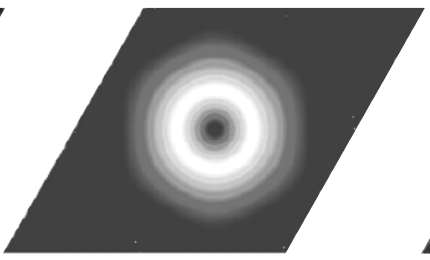

$\rho=0.0625, E / 8 \pi=1.082$

д

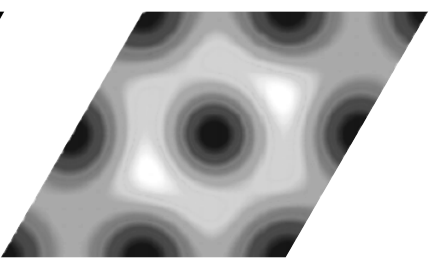

$\rho=0.25, E / 8 \pi=1.087$

B

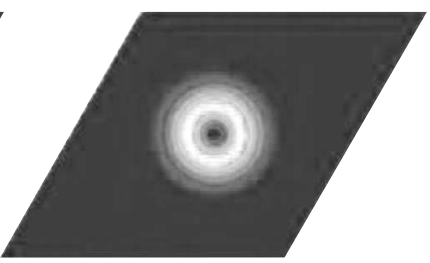

$\rho=0.03125, E / 8 \pi=1.084$

e

Рис. 7. Скирмион с зарядом два в общем случае при $\mu^{2}=0.1$ и $\kappa^{2}=0.03$ : на контурных графиках приведены зарядовые плотности для конфигураций с минимальной энергией на шестиугольной решетке при различных плотностях (энергетически предпочтительной оказывается плотность $\rho \approx 0.14$ ).

производные к нулю:

$$
\begin{aligned}
& \frac{\partial E}{\partial \gamma}=\frac{1}{2 s \cos ^{2} \gamma}\left(\sin \gamma\left(\mathcal{E}^{y y}+s^{2} \mathcal{E}^{x x}\right)-2 s \mathcal{E}^{x y}\right)=0, \\
& \frac{\partial E}{\partial s}=\frac{1}{2 s^{2} \cos \gamma}\left(\mathcal{E}^{y y}-s^{2} \mathcal{E}^{x x}\right)=0
\end{aligned}
$$

где $\mathcal{E}^{i j}=\int_{\mathbb{T}^{2}} d x d y\left(\partial_{i} \phi \cdot \partial_{j} \phi\right)$ и $i, j \in\{x, y\}$. Решение этих уравнений относительно $\gamma$ и $s$ дает

$$
s=\sqrt{\frac{\mathcal{E}^{y y}}{\mathcal{E}^{x x}}}, \quad \sin \gamma=\frac{\mathcal{E}^{x y}}{\sqrt{\mathcal{E}^{x x} \mathcal{E}^{y y}}} .
$$

Подставляя эти выражения в функционал энергии (7), получаем "редуцированный" функционал

$$
E=\sqrt{\mathcal{E}^{x x} \mathcal{E}^{y y}-\left(\mathcal{E}^{x y}\right)^{2}}+\frac{\kappa^{2} \rho}{2 B} \mathcal{E}_{\mathrm{Sk}}+\frac{\mu^{2} B}{\rho} \mathcal{E}_{\mathrm{pot}},
$$

где

$$
\mathcal{E}_{\mathrm{Sk}}=\int_{\mathbb{T}^{2}} d x d y\left(\partial_{x} \phi \times \partial_{y} \phi\right)^{2}, \quad \mathcal{E}_{\mathrm{pot}}=\int_{\mathbb{T}^{2}} d x d y\left(1-\phi_{3}\right)
$$

- энергия Скирма и потенциальная энергия соответственно. Далее, когда и $\gamma$, и $s$ исключены из получившегося выражения, а условия их оптимизации встроены в функционал, мы выполняем численную минимизацию. Заметим, однако, что представленную выше процедуру следует воспринимать с осторожностью. Дело в том что 
условия (10) для $\gamma$ и $s$ в действительности являются только условиями экстремума и могут давать максимум или седловую точку. Поэтому важно подтвердить любые результаты, полученные с использованием данного метода, сравнивая их с соответствующими результатами, полученными методом, описанным в разделе 4 . Весьма отрадно, что численная минимизация редуцированного функционала (12) дает $\sin \gamma=0.498(\gamma \approx \pi / 6)$ и $s=1$ (и в случае Скирма, и в общем случае), что подтверждает результаты, представленные в предыдущем разделе.

В общем случае $(\mu \neq 0)$ функционал энергии (12) можно дифференцировать по скирмионной плотности $\rho$, что дает оптимальную плотность, для которой энергия скирмиона минимальна. Дифференцирование по $\rho$ и подстановка полученного выражения в функционал энергии приводит к функционалу

$$
E=\sqrt{\mathcal{E}^{x x} \mathcal{E}^{y y}-\left(\mathcal{E}^{x y}\right)^{2}}+\kappa \mu \sqrt{2 \mathcal{E}_{\mathrm{Sk}} \mathcal{E}_{\mathrm{pot}}}
$$

Численная минимизация приведенного выражения при $\kappa^{2}=0.03$ и различных значениях $\mu\left(0.1 \leqslant \mu^{2} \leqslant 10\right)$ дает шестиугольную конфигурацию, как и в случае Скирма. В частности, при $\mu^{2}=0.1$ оптимальная плотность оказывается равной $\rho \approx 0.14$, что согласуется с результатами из п. 5.3.

\section{7. ИТОГИ И ЗАМЕЧАНИЯ О ДАЛЬНЕЙШИХ ИССЛЕДОВАНИЯХ}

Мы исследовали решеточную структуру бэйби-скирмионов в двумерном пространстве путем поиска при периодических граничных условиях конфигураций с минимальной энергией, образованных бэйби-скирмионами, расположенными внутри единичной ячейки в форме параллелограмма. В чистом $O(3)$-случае (когда и скирмионный, и потенциальный члены отсутствуют) мы проверили, что нет предпочтительных решеток, в которых скирмионы самоупорядочиваются, поскольку все конфигурации параллелограммов дают одну и ту же минимальную энергию. Эта энергия отвечает минимальной величине в ограничении на энергию, задаваемом неравенством (6).

В случае Скирма, т.е. без потенциального члена, результаты иные. При любой фиксированной скирмионной плотности параллелограммы, для которых скирмионная энергия минимальна, образуют шестиугольную решетку $(s=1$ и $\gamma=\pi / 6)$. В частности, шестиугольная решетка имеет более низкую энергию, чем конфигурация из четырех полускирмионов на прямоугольной решетке. Например, при $\rho=2$ для шестиугольной решетки $E=1.433$, для прямоугольной решетки $E=1.446$.

Шестиугольная структура оказывается энергетически наиболее предпочтительной также и в общем случае, когда имеются и скирмионный, и потенциальный члены. В этом случае, однако, модель не является масштабно-инвариантной и скирмион имеет определенный размер. Это приводит к существованию точки фазового перехода, зависящей от плотности, и к появлению оптимальной плотности, при которой полная энергия скирмиона минимальна. Это аналогично скирмионному поведению в трехмерной модели Скирма, где скирмионы также обладают определенным размером [22]. 
Как уже отмечалось выше, обнаруженная в настоящей работе специальная роль шестиугольных решеток не уникальна для бэйби-модели Скирма: она возникает и в других солитонных моделях. В контексте моделей Скирма существование шестиугольной двумерной структуры трехмерных скирмионов было обнаружено в работе [32], где отмечено, что оптимальная с энергетической точки зрения бесконечная плоская структура трехмерных скирмионов представляет собой решетку, напоминающую структуру листа графита - термодинамически наиболее устойчивой формы углерода (см. книгу [10]). Другими примерами, в которых проявляется шестиугольньная структура, являются вихри Гинзбурга-Ландау, о которых известно, что в шестиугольной конфигурации они имеют более низкую энергию, чем в конфигурации квадратной решетки [31]. Таким образом, не следует удивляться тому, что шестиугольная структура оказывается наиболее предпочтительной в бэйби-модели Скирма.

Бэйби-скирмионы определенного вида также возникают в системах с квантовым эффектом Холла, где они появляются как низкоэнергетические возбуждения основного состояния вблизи ферромагнитных чисел заполнения (особенно вблизи 1 и 1/3) [5]. Уже указывалось [34], что это состояние содержит конечную плотность скирмионов, в работе [6] шестиугольная конфигурация была предложена в качестве кандидата на их решеточную структуру. Наши результаты могут служить дополнительным свидетельством в этом направлении, хотя здесь и требуется более подробный анализ.

Проведенные нами исследования также дают основание поставить некоторые интересные вопросы: как динамические свойства бэйби-скирмионов на шестиугольной решетке отличаются от их поведения на обычной прямоугольной решетке, как ведут себя скирмионы при ненулевой температуре? Можно также поинтересоваться и обобщением этих результатов на трехмерный случай, коль скоро будет выполнено систематическое исследование, подобное проведенному в настоящей работе. Действительно ли среди всех решеток в форме параллелепипеда гранецентрированная кубическая решетка является кристаллической структурой трехмерных скирмионов с минимальной энергией? Если нет, то какова будет структура с минимальной энергией? Как эти результаты изменятся в присутствии массового члена? Мы надеемся ответить на эти вопросы в дальнейших исследованиях.

Благодарности. Данная работа частично поддержана со стороны Israel Science Foundation под управлением Israel Academy of Sciences and Humanities.

\section{Список литературы}

[1] T. H. R. Skyrme, Proc. R. Soc. Lond. Ser. A, 260:1300 (1961), 127-138; Nucl. Phys., 31 (1962), 556-569.

[2] G. S. Adkins, C. R. Nappi, E. Witten, Nucl. Phys. B, 228:3 (1983), 552-566.

[3] B. M. A. G. Piette, B. J. Schoers, W. J. Zakrzewski, Z. Phys. C, 65:1 (1995), 165-174.

[4] А. А. Белавин, А. М. Поляков, Писъма в ЖЭТФ, 22:10 (1975), 503-506.

[5] S. L. Sondhi, A. Karlhede, S.A. Kivelson, E. H. Rezayi, Phys. Rev. B, 47:24 (1993), 16419-16426. 
[6] N. R. Walet, T. Weidig, Full 2D numerical study of the quantum Hall skyrme crystal, arXiv: cond-mat/0106157.

[7] S. M. Girvin, "The quantum Hall effect: novel excitations and broken symmetries", Topological Aspects of Low Dimensional Systems, eds. A. Comtet, J. Jolicoeur, S. Ouvry, F. David, Springer, Berlin, 1999, 53-175.

[8] Z. F. Ezawa, Quantum Hall Effects: Field Theoretical Approach and Related Topics, World Sci., Singapore, 2000.

[9] D.-H. Lee, C. L. Kane, Phys. Rev. Lett., 64:12 (1990), 1313-1317.

[10] N. S. Manton, P. M. Sutcliffe, Topological Solitons, Cambridge Monogr. Math. Phys., Cambridge Univ. Press, Cambridge, 2004.

[11] R. A. Leese, M. Peyrard, W. J. Zakrzewski, Nonlinearity, 3:3 (1990), 773-807.

[12] B. Piette, W. J. Zakrzewski, Chaos Solitons Fractals, 5:12 (1995), 2495-2508.

[13] P. M. Sutcliffe, Nonlinearity, 4:4 (1991), 1109-1121.

[14] B. M. A. G. Piette, B. J. Schoers, W. J. Zakrzewski, Nucl. Phys. B, 439:1-2 (1995), 205-235.

[15] T. Weidig, Nonlinearity, 12:6 (1999), 1489-1503.

[16] P. Eslami, M. Sarbishaei, W. J. Zakrzewski, Nonlinearity, 13:5 (2000), 1867-1881.

[17] I. Hen, M. Karliner, Nonlinearity, 113:3 (2008), 399-408.

[18] I. Hen, M. Karliner, Phys. Rev. D, 77:5 (2008), 054009.

[19] E. Braaten, S. Townsend, L. Carson, Phys. Lett. B, 235:1-2 (1990), 147-152.

[20] R. A. Battye, P. M. Sutcliffe, Phys. Rev. Lett., 79:3 (1997), 363-366.

[21] N. R. Walet, Nucl. Phys. A, 606:3-4 (1996), 429-458.

[22] I. Klebanov, Nucl. Phys. B, 262:1 (1985), 133-143.

[23] A. S. Goldhaber, N. S. Manton, Phys. Lett. B, 198:2 (1987), 231-234.

[24] A. D. Jackson, J. J. M. Verbaarschot, Nucl. Phys. A, 484:3-4 (1988), 419-431.

[25] M. Kugler, S. Shtrikman, Phys. Lett. B, 208:3-4 (1988), 491-494.

[26] L. Castellejo, P.S. J. Jones, A. D. Jackson, J. J. M. Verbaarschot, Nucl. Phys. A, 501:4 (1989), 801-812.

[27] O. Schwindt, N. R. Walet, Europhys. Lett., 55:5 (2001), 633-639.

[28] R. S. Ward, Nonlinearity, 17:3 (2004), 1033-1040.

[29] R. J. Cova, W. J. Zakrzewski, Nonlinearity, 10:5 (1997), 1305-1317; Eur. Phys. J. B, 15:4 (2000), 673-678; Rev. Mexicana Fis., 50:5 (2005), 527-535.

[30] J. Bamberg, G. Cairns, D. Kilminster, Amer. Math. Monthly, 110:3 (2003), 202-209.

[31] W. H. Kleiner, L. M. Roth, S. H. Antler, Phys. Rev. A, 133:5A (1964), A1226-A1227.

[32] R. A. Battye, P. M. Sutcliffe, Phys. Lett. B, 416:3-4 (1998), 385-391.

[33] M. Hale, O. Schwindt, T. Weidig, Phys. Rev. E, 62:3 (2000), 4333-4346.

[34] L. Brey, H. A. Fertig, R. Côté, A. H. MacDonald, Phys. Rev. Lett., 75:13 (1995), 2562-2565. 